\title{
Atom Probe Tomography and analytical Scanning Transmission Electron Microscopy of Rapid Solidification Microstructures in Al-Cu Alloy Thin Films
}

\author{
Jörg M.K. Wiezorek ${ }^{1}$, Kai W. Zweiacker ${ }^{1,2}$, Can Liu ${ }^{1}$, Isabelle Martin ${ }^{3}$, Ty. J. Prosa ${ }^{3}$, David J. Larson ${ }^{3}$ \\ 1. Department of Mechanical Engineering and Materials Science, Swanson School of Engineering, \\ University of Pittsburgh, Pittsburgh, PA, USA. \\ 2. Now: EMPA, Swiss Federal Laboratories for Materials Science and Technology, Dübendorf, \\ Switzerland. \\ 3. CAMECA Instruments Inc., Madison, WI, USA.
}

Laser processing of alloys results in formation of rapid solidification (RS) microstructures that can exhibit refined length scale, extreme solute segregation, formation of meta-stable phases, and unusual textures [1]. Recent nano-scale spatio-temporal resolution in situ dynamical transmission electron microscopy (DTEM) studies of pulsed laser (PL) induced RS in multi-component alloys of hypoeutectic $\mathrm{Al}-\mathrm{Cu}$ thin film specimens have identified characteristic morphological modulations of the multi-phase microstructures with solidification interface velocity [1, 2]. Elucidating constitutional effects during RS crystal growth in multi-component systems is of fundamental importance in understanding laser-assisted materials processing [1-5]. However, due to the nano-scale of the resulting microstructural features, acquiring quantitative analytical data with the appropriate spatial-resolution is challenging with scanning and transmission electron microscopes (SEM and TEM). A promising alternative analytical technique is atom probe tomography (APT). APT offers sub-nanometer spatial resolution, and provides statistically significant representative data sets for elemental composition mapping, but is limited to relatively small analytical volumes. The current work presents complementary aberration-corrected scanning TEM (STEM) based energy dispersive X-ray spectroscopy (EDXS) mapping to study constitutional effects in RS microstructure evolution of hypo-eutectic Al-Cu alloys.

$\mathrm{Al}-\mathrm{Cu}$ thin film alloy RS microstructures were generated by PL irradiation of the electron transparent regions of silicon nitride membrane supported windows $[1,2,6]$. APT specimens were prepared using focused ion beam methods [7] from regions at the perimeter of the PL induced melt-pool ( $25 \mu \mathrm{m}$ by $\sim 230 \mu \mathrm{m}$, dotted line Fig. 1a). High RS interface velocities, up to $\sim 2.0 \mathrm{~m} / \mathrm{s}$, generate four distinct morphological zones: A heat affected zone (HAZ, Zone 1), a narrow transition region (Zone 2) with elongated $\alpha$-Al grains, the RS crystal growth region with columnar morphology cellular two-phase growth (Zone 3a), and the banded-morphology growth region (Zone 3b) (Fig. 1b) [2, 6]. Under equilibrium conditions hypoeutectic $\mathrm{Al}-\mathrm{Cu}$ contains the face-centered cubic $\alpha$-Al matrix (up to $2.6 \mathrm{at} \% \mathrm{Cu})$ and tetragonal $\theta-\mathrm{Al}_{2} \mathrm{Cu}(\sim 33 \mathrm{at} \% \mathrm{Cu})$ phase. The DF-STEM image inset in Figure 1b of the melt-pool edge shows the columnar growth zone (Zone 3a) with discontinuous fine-scale copper enriched metastable $\mathrm{Al}_{2} \mathrm{Cu}\left(\theta^{\prime}\right)$ phase, the transition region with elongated $\alpha-\mathrm{Al}$ grains (Zone 2) and the $\mathrm{Cu}$-enriched continuous network of $\theta-\mathrm{Al}_{2} \mathrm{Cu}$ phase in Zone 1 [6]. $\mathrm{Cu} \%$-iso-surfaces and proxigrams for APT specimen M08 (Zone 1) and M05 (Zone 2 / Zone 3a) reveal clearly two distinct phases, corresponding to supersaturated $\alpha-\mathrm{Al}(\sim 4 \mathrm{at} \% \mathrm{Cu})$ and $\mathrm{Al}_{2} \mathrm{Cu}$ related phase $(\sim 33 \mathrm{at} \% \mathrm{Cu}$ for Zone 1 and $\sim 42 \mathrm{at} \% \mathrm{Cu}$ for Zone 2 / Zone 3a), respectively (Figs. 2a, 2b). APT and STEM EDXS analyses showed nearly identical $\mathrm{Cu} \%$ for the $\alpha$-Al phase in Zones 1, 2 and 3a (Fig. 2). STEM EDXS measurements tended to underestimate the $\mathrm{Cu}$-content of the $\mathrm{Al}_{2} \mathrm{Cu}$ related phase, since this minority constituent is typically embedded in the majority matrix phase of $\alpha$-Al. However, STEM EDXS analyses of the continuous layers of $\mathrm{Cu}$-enriched phase in Zone 2 (Figs. 2c, 2d) showed enhanced $\mathrm{Cu} \%$ with on average 
$39.7 \mathrm{at} \% \mathrm{Cu}$. In the Zone 2 a rapid acceleration of the previously stagnant solid-liquid interface to $\sim 0.8 \mathrm{~m} / \mathrm{s}$ occurs after a significant incubation time following PL melting [6]. The composition variations in the $\alpha$-Al phase and the $\mathrm{Cu}$-enriched $\mathrm{Al}_{2} \mathrm{Cu}$ related phase in Zones 1,2 and 3a, inclusive of the local increase beyond $33 \mathrm{at} \% \mathrm{Cu}$ expected for stoichiometric $\mathrm{Al}_{2} \mathrm{Cu}$, represent clear deviations from equilibrium crystal growth. The Al-Cu alloy composition analyses by APT and STEM will be discussed and correlated with the solid-liquid interface velocity evolution during the PL induced RS [8].

\section{References:}

[1] J.E. Kline and J.P. Leonard, Applied Physics Letters 86 (2005), p. 201902.

[2] J.T. McKeown et al, Acta Materialia 65 (2014), p. 56.

[3] S.C. Gill et al, Acta Metallurgica Materiala 40(1992), p. 2895.

[4] S.C. Gill and W. Kurz, Acta Metallurgica Materiala 41(1993), p.3 563.

[5] W. Kurz and P. Gilgien, Materials Science and Engineering A 178(1994), p. 171.

[6] J.T. McKeown et al, JOM 68 (2016), p. 985.

[7] D. J. Larson et al., "Local Electrode Atom Probe Tomography”, (Springer, New York) (2013).

[8] The authors acknowledge funding from the National Science Foundation, Grants NSF-1105757 and NSF-1607922.

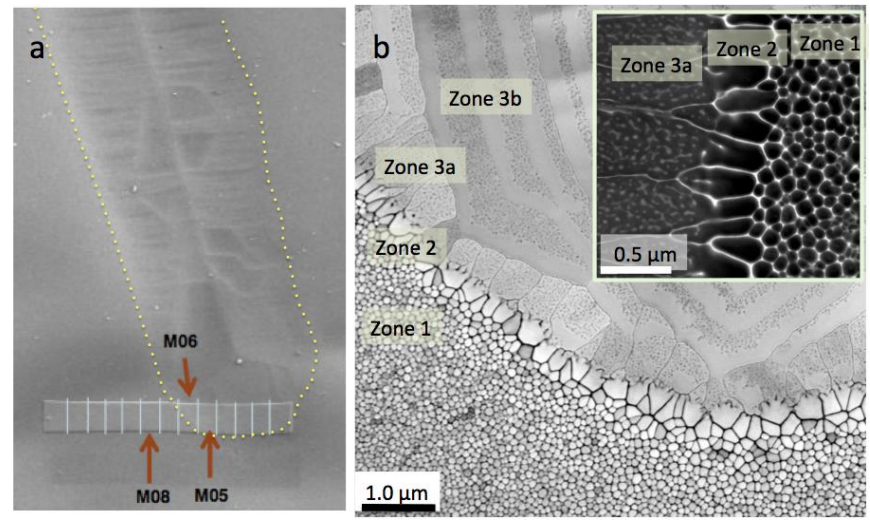

Figure 1. a) SEM secondary electron image of $\mathrm{RS}$ region in $\mathrm{Al}-10 \mathrm{Cu}$ alloy thin film (inside dotted outline) and location for APT specimen extractions, e.g. M08 (Fig. 2a) and M05 (Fig. 2b), b) STEM BF and as inset enlarged detail STEM HAADF of the RS microstructure zones: Zone 1, Zone 2, Zone 3a and Zone 3b.
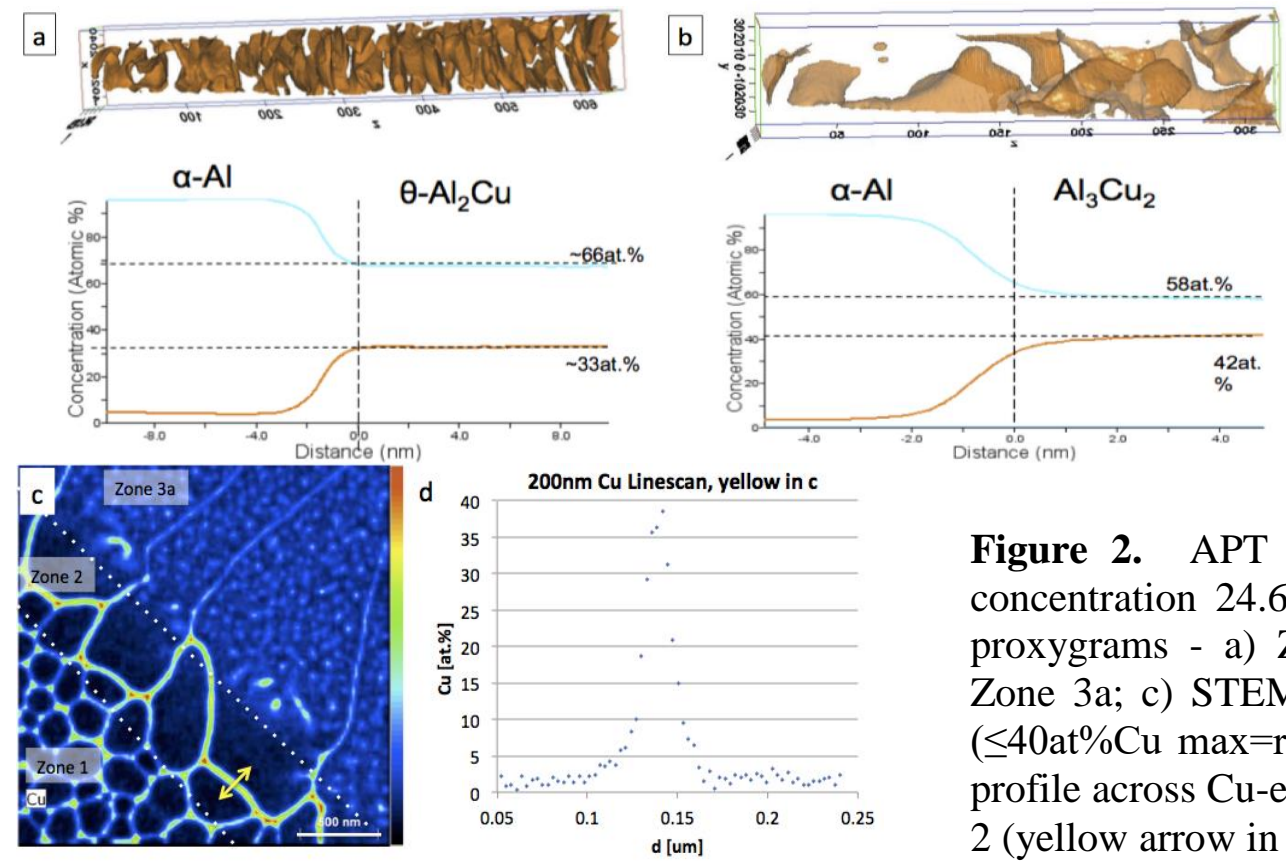

Figure 2. APT $\mathrm{Cu}$ ion map for isoconcentration $24.6 \mathrm{at} \% \mathrm{Cu}$ and associated proxygrams - a) Zone 1, b) Zone 2 to Zone 3a; c) STEM EDXS Cu heat map ( $\leq 40 \mathrm{at} \% \mathrm{Cu} \max =\mathrm{red})$; d) $\mathrm{Cu}$ EDXS line profile across $\mathrm{Cu}$-enriched region in Zone 2 (yellow arrow in c)). 